\title{
IMAGE PROCESSING OF TEMPERATURE FIELDS FROM INFRARED TERMOGRAPHY OF MICRO-MIXERS WITH POLYMERIC SUBSTRATES
}

\author{
J. M. Costa Junior ${ }^{\mathrm{a}}$, \\ C. P. Naveira-Cotta ${ }^{a}$, \\ and O. Fudym \\ ${ }^{a}$ Universidade Federal do Rio de Janeiro \\ Laboratory of Microfluidics and Microsystems, \\ LabMEMS \\ Mechanical Engineering Dept. \\ Escola Politécnica \& COPPE \\ Cidade Universitária, CEP. 68503, \\ Rio de Janeiro, RJ, Brazil \\ carolina@mecanica.coppe.ufrj.br \\ ${ }^{\mathrm{b}}$ Université de Toulouse, Mines Albi \\ CNRS,Centre RAPSODEE \\ Campus Jarlard \\ F. 81013 Albi cedex 09, Franc \\ Received: October 25, 2013 \\ Revised: November 20, 2013 \\ Accepted: December 20, 2013
}

\section{ABSTRACT}

The present work deals with the image processing and thermal analysis of micro-mixers from the data provided by an infrared camera thermography system. The micro-mixers are prepared by photolitography on a polymeric substrate and the camera employed is the FLIR SC645 with the proprietary software ThermaCam Researcher Pro v2.10. The thermal analysis is aimed at understanding the direct contact heat transfer between two fluid streams and the polymeric substrate at different inlet temperatures and mass flow rates, within mixers of various geometric configurations. Infrared thermography is thus employed to measure the external wall temperatures fields along the mixer length. Water at different inlet temperatures has been used as the working fluid in all cases and the mass flow rates of the two streams have been imposed through independent syringe pumps. The image processing and analysis of the experimental results show the basic qualitative and quantitative features of the heat transfer phenomena and indicates that a conjugated heat transfer formulation of the micro-mixer structure should be pursued for accurate quantitative analysis in theoretical predictions.

Keywords: micro-mixers, infrared thermography, image processing, microchannel, photolithography.

\section{NOMENCLATURE}

$\mathrm{f}_{\mathrm{y}}$ dimension of the image pixel in the transverse direction, $\mathrm{mm}$

$\mathrm{f}_{\mathrm{z}}$ dimension of the image pixel in the axial direction, $\mathrm{mm}$

$\mathrm{Pe} \quad$ Peclet number

$\mathrm{R}$ dimensionless radial coordinate

Re Reynolds number in mixing channel

$\mathrm{T}_{\text {exp }}$ experimental temperature, ${ }^{\circ} \mathrm{C}$

U(R) dimensionless velocity

$\mathrm{u}_{\mathrm{m}} \quad$ average velocity, $\mathrm{m} / \mathrm{s}$

$\mathrm{y}$ transverse coordinates

$\mathrm{z} \quad$ axial coordinates

\section{Greek symbols}

$\theta_{\mathrm{f}} \quad$ dimensionless temperature of the fluid

$\varphi$ dimensionless longitudinal coordinate

\section{Subscripts \\ exp experimental \\ f fluid \\ m average}

$$
\begin{array}{ll}
\text { y transversal coordinate } \\
\mathrm{z}
\end{array}
$$

\section{INTRODUCTION}

Micro-mixers are one of the essential components required in the assembly of various types of MEMS (Micro-Electro-Mechanical Systems) due to the need of enhancing mixing effects in light of the laminar nature and predominant diffusive mixing of the more usual flows in microfluidic devices. The micro-mixers, by presenting small dimensions, become appropriate in controlling the mixture of reagents under very low Reynolds number and laminar flows. Besides using small amounts of reagents, they also allow to easily, safely and quickly test a great number of reaction configurations (Tabeling, 2003). Thus, micro-mixers are essential components in microfluidic systems, of great interest to both industry and academy within numerous applications, such as chemical and biochemical analysis, monitoring and control of processes, automation of operations in petroleum reservoirs, among others. 
The studies about the theoretical principles and applications of these devices are fairly recent and have attracted the interest of many researchers, mainly in relation to the fluid flow and heat/mass transfer of mixing streams in micro-channels. For instance, Engler et al. (2004) demonstrated by numerical simulations and experiments the effects of vorticity in a static micro-mixer of rectangular section and concluded that the increase of the effect of vorticity can improve the quality of mixing and that the development of vortices does not depend only on the Reynolds number, but also highly on the channel geometry. Pradere et al. (2004) performed an analysis of the temperature fields in micro-reactors using infrared thermography and image processing methods, with the aim of proposing a new estimation method in order to access the source term distribution inside the channel, which consisted in inverting the pseudo-temperature fields inside and outside the channel using simplified models. Pohar and Plazl (2009) reported the benefits and the significant advances in the use of micro-reactors in existing manufacturing processes or in their complete substitution, since with micro-systems there is a better control of the temperature due to the resulting high rates of heat and/or mass transfer and achievement of high reaction rates and consequent reduction of the reaction time. $\mathrm{Xu}$ et al. (2010) experimentally and numerically investigated the flow and mixing features of two miscible fluid streams in a T-shaped micro-channel.

The study of the thermal behavior of mixtures is also of fundamental importance once the information of the temperature within the devices serves as a tracer to the monitoring of the process. Then, it is necessary to use reliable measurement techniques, capable of precisely providing the variations of temperature along the micro-mixers. In this context, the use of the infrared thermography technique provides non- intrusive temperature measurements with high temporal resolution and, nowadays, progressively improving spatial resolution.

The main objective of the present work is the qualitative and quantitative thermal analysis of micro-mixers of various geometric configurations, which work with direct contact heat transfer between two fluid streams at different inlet temperatures, using infrared thermography to compare various cases with different temperatures and mass flow rates of the two streams. We also present an estimation of the flow Peclet number through the pseudotemperature field outside the channel, processing the infrared camera images in a micro-mixer made of polymeric substrate.

The fabrication technique of the micro-devices based on photolitography used in this work was developed by Fernandes and Ferreira (2006). It uses a photoresist based in the urethane and acrylate oligomers (UA) and was described in detail in that work.

\section{PHOTOLITOGRAPHY FABRICATION}

Photolitography was used to manufature the micro-mixers. The energy source was ultraviolet (U.V.) light. The U.V. light was used to transfer a geometric pattern from a photomask to a lightsensitive chemical "photoresist", or simply "resist", via a selective illumination of this material. This selective exposure is performed using a photomask, between the light source and the photoresist material. The photomask in the case of the photoligraphy is transparent to U.V. light on its light areas and blocks the U.V. light on its dark areas.

In general, the micro-fabrication process by photolithography can be described through four main steps.

- $\quad$ The first step consists in the preparation of the photoresist and in its deposition;

- The second step of the process consists in the selective exposure of the photoresist by the U.V. light;

- The third step consists in removing the unexposed polymer using a special solution, called "developer";

- The fourth step consists of sealing the micro-system so that it supports operating conditions without the occurrence of leaks.

Figure 1 schematically shows the four steps of the micro-fabrication process via photolithography.

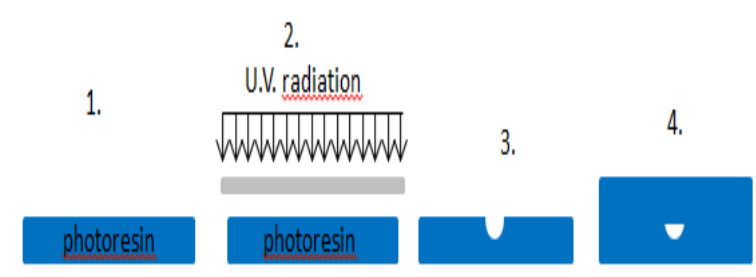

Figure 1. Scheme of the micro-fabrication process by photolitography.

Figure 2 shows the step of deposition of the photoresist (a viscous liquid solution) in a region bounded by a frame, after which the whole set is exposed to U.V. light in a photoexposer machine as presented in Figure 3. Exposure to the UV light causes the resist to become polymerized, and more difficult to dissolve. Therefore, the negative resist remains on the surface wherever it is exposed, and the developer solution will remove only the unexposed portions. In the present work the photomasks used in the photolithography process contain the inverse (or photographic "negative") of the pattern to be transferred and were made using overhead projector transparencies (CG7031S) and the drawings of the micro-channel were made using the graphic software AutoCAD 2008 (AutoDesk ${ }^{\circledR}$ ). The photomasks were printed using a laser printer (HP LaserJet P1005) with a resolution of 1200 dpi. 


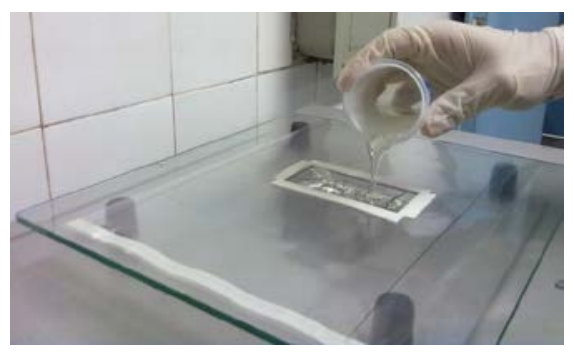

Figure 2. Deposition of the photoresist (step 1 of the micro-manufacturing process).

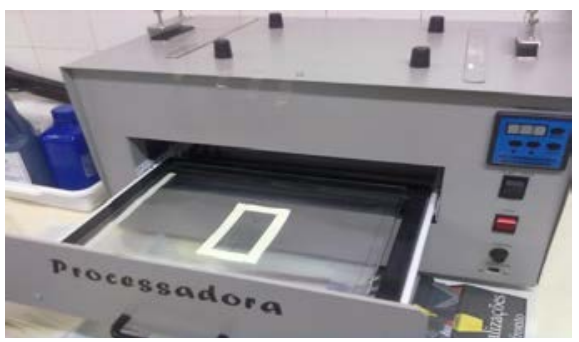

Figure 3. Photoexposer machine (step 2 in the micro-manufacturing process).

The exposure time determines the depth of channel that will be manufactured, since the greater the exposure time further polymerization will suffer the photoresist so that the channels are less deep. However, the exposure time may vary according to the polymer used and the photoexposer machine employed so that one must make a calibration of the process until reaching the desired dimensions.

The next step in the micro-manufacturing process is to remove the negative resist that remains on the surface wherever it is exposed. In the present study, this developer solution is a mixture of water and detergent. Figure 4 shows the development process using a ultrasound bath of water and detergent and after that it is used a jet of solvent directly onto the channel to assist in the removal of excess polymer which accumulates in the region.

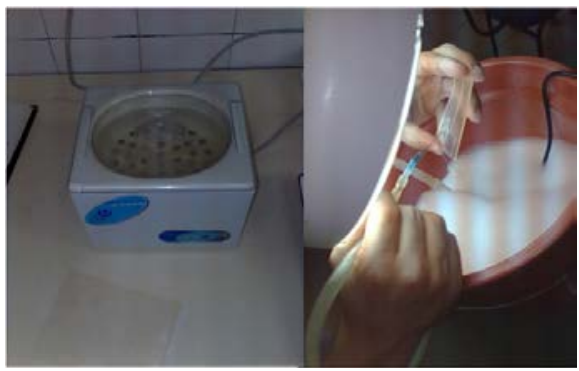

Figure 4. Development process (step 3 in the micromanufacturing process).

For the sealing step, a lid is manufactured for the micro-system, similarly to was shown previously. However, in the manufacturing of this cover, the photomask is not used, so that all the polymer is exposed to UV light and therefore polymerized The sealing process of these two parts (cover and base) is performed by making the simple union of the base (containing the micro-channel) and the lid and reexposing the assembly (cover + base) to U.V. light after imposing a flow of argon gas in order to provide an oxygen-free atmosphere around the substrate. Thus, the excess of polymer which was not completely removed in the development step, and still is on the surface of the lid and of the base, undergoes polymerization providing a direct chemical bond (i.e., intermolecular bond without intermediate layers) between the cover and the base. Figure 5 illustrates the process of sealing the microsystem.

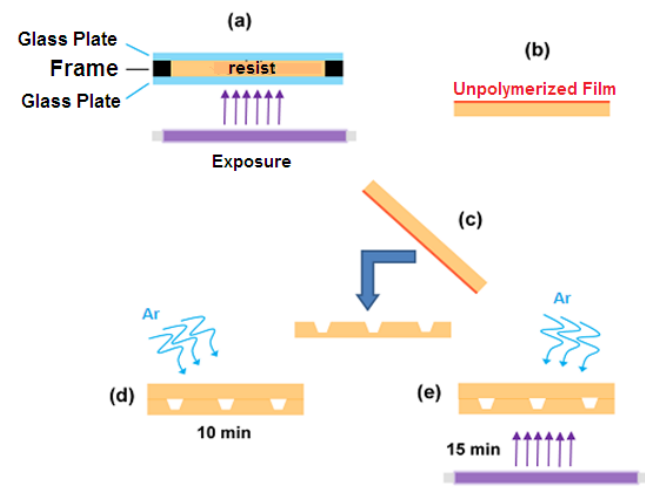

Figure 5. Scheme of the sealing procedure (Fonseca, 2008).

Finally, the system is connected to the outside world through hoses and commercial hypodermic needles as shown in Figure 6.

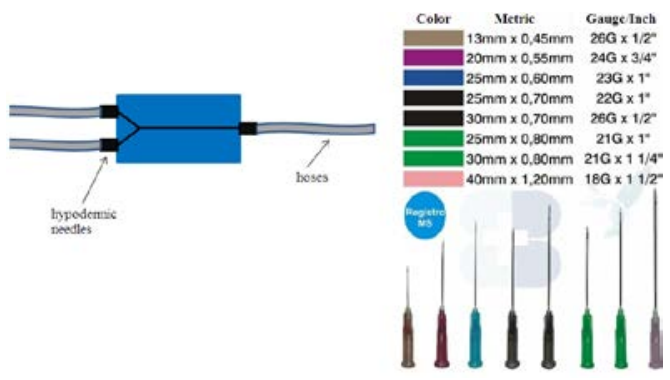

Figure 6. Connecting the system to the outside world.

\section{EXPERIMENTAL SETUP}

The experimental setup presented in Figure 7 employs temperature measurements obtained from the infrared camera FLIR SC645, a high performance infrared system with $640 \times 480$ pixels. During the experiments the working distance between object and lens was $28 \mathrm{~cm}$, for all micro-mixer configurations.

The main components of the setup are marked on Figure 7 as a) the micro-system; b) the dual syringe pumps for controlled and independent flow rates; c) the data acquisition system (Agilent 34970- 
A); d) the microcomputer for data acquisition from the thermocouples; e) the precision scale, to obtain the total mass flow rate at the outlet of the micromixer; f) infrared camera FLIR SC645. Three thermocouples were used to measure the bulk temperatures in the fluid, two of them located at each entry of the micro-mixer and one located at the outlet of the mixing micro-channel. A fourth one was used to measure the ambient temperature.

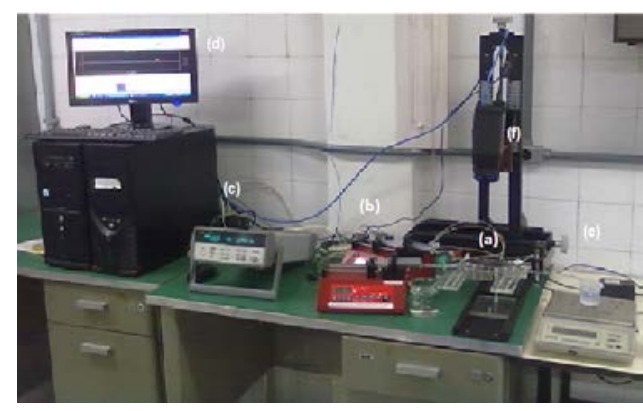

Figure 7. Experimental setup.

The three micro-mixers used in the experiments conducted in this study consist of straight, sinuous and zig-zag configurations, fabricated by the photolithography process, described in detail in the previous section. All these micro-mixers have access channels arranged in a Y-shape and are contained in a polymeric chip of $8 \times 4 \mathrm{~cm}$, horizontally positioned.

The straight micro-mixer consists of a straight mixing channel that has a diameter of approximately $450 \mu \mathrm{m}$ and a mixing length of $6 \mathrm{~cm}$. Figure 8 shows the straight micro-mixer and the microscopic images obtained by optical microscopy.

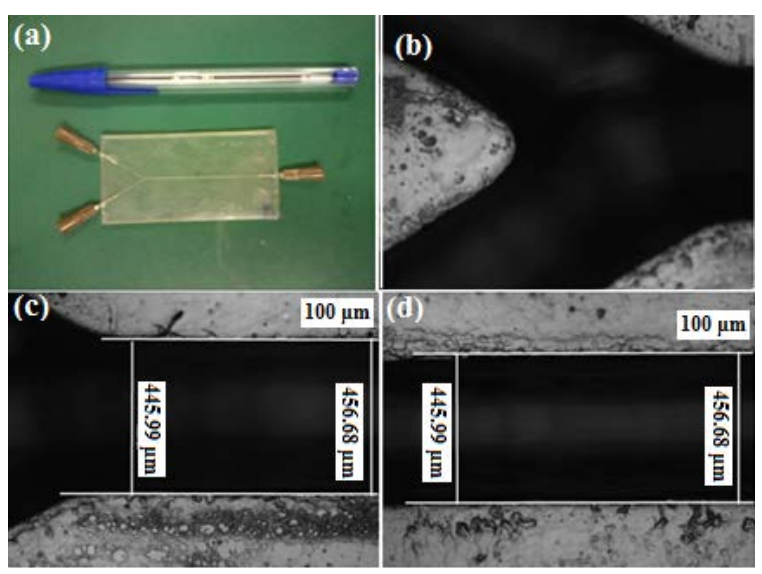

Figure 8. Straight micro-mixer and microscopic images.

The sinuous micro-mixer consists of a mixing channel in periodic steps which has a diameter of approximately $300 \mu \mathrm{m}$ and a mixing length of about $26 \mathrm{~cm}$. Figure 9 shows the sinuous micro-mixer and its microscopic images.

The zig-zag micro-mixer consists of a mixing channel with periodic triangular configuration, with a diameter of approximately $300 \mu \mathrm{m}$ and a mixing length of $12.8 \mathrm{~cm}$. Figure 10 shows the zig-zag micro-mixer and its microscopic images.

The present analysis deals with a process of thermal mixing via direct contact heat transfer between two water currents at different inlet temperatures, one below and the other above room temperature, and equal or different mass flow rates, imposed through the two independent syringe pumps. The experimental procedure is initiated by prescribing the flow rates at the syringe pumps and setting the parameter values relative to the infrared camera, such as the emissivity of the object, the distance between the object and the camera, relative humidity and ambient temperature. The data acquisition system is started and after a certain number of preliminary measurements to allow for averaging the initial conditions, the pumps switches are turned on to start the two fluid streams. The temperatures variation may be followed through the computer monitoring of both the infrared camera and the thermocouples acquisitions.
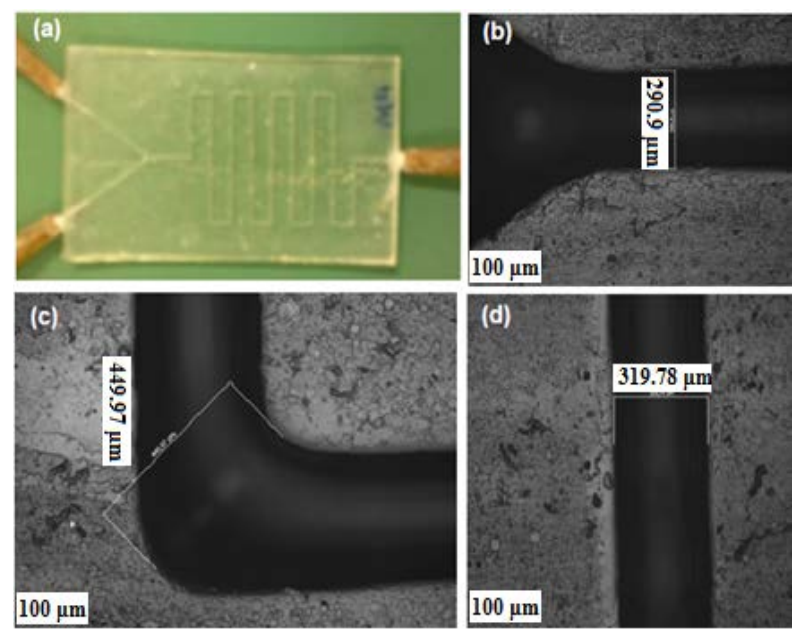

Figure 9. Sinuous micro-mixer and microscopic images.
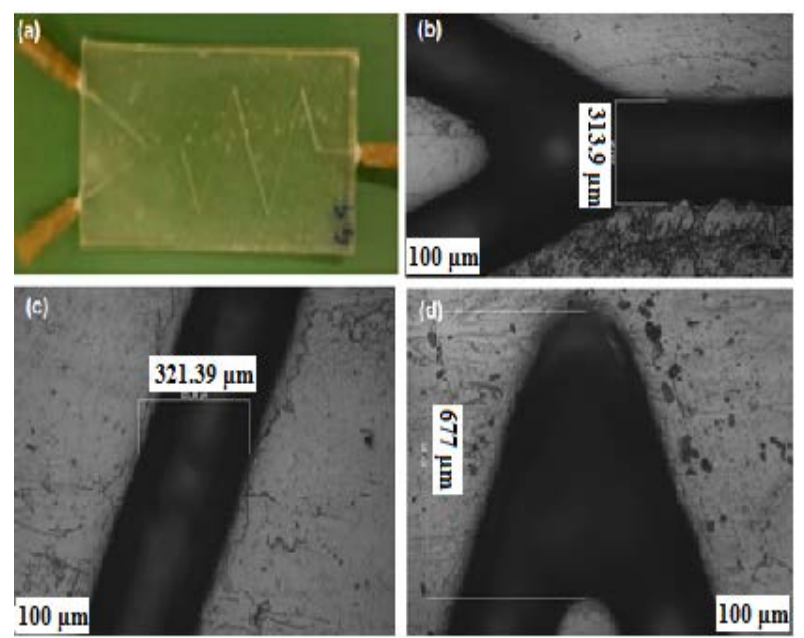

Figure 10. Zig-zag micro-mixer and microscopic images. 


\section{EXPERIMENTAL CASES}

In this paper we analyzed three experimental cases for each of the three configurations of micromixer described in the previous section. All cases performed in this work are shown in Table1 and to facilitate the understanding, the input channel through which heated water flows is called "Hot Entry" and the input channel that flows the cold water is called "Cold Entry". Case 1 refers to the experiment performed on the straight micro-mixer when it was used equal volumetric flow rates in both channels (flow rate $1.0 \mathrm{ml} / \mathrm{min}$ ) and the working fluids at equal temperatures (both streams with heated water). Case 2 refers to the experiment with the sinuous micro-mixer where unequal temperatures and flow rates were employed in the two access channels, with a flow rate of $1.0 \mathrm{ml} / \mathrm{min}$ in the "Cold Entry" and $2.0 \mathrm{ml} / \mathrm{min}$ in the "Hot Entry". Case 3 refers to the experiment conducted in the Zig-Zag micro-mixer and settings for flow rates and temperatures at the entrance of the access channels are analogous to those of the sinuous micro-mixer configuration (Case 2).

Table 1. Experimental Cases.

\begin{tabular}{|c|c|c|c|c|c|}
\hline case & $\begin{array}{c}\dot{m}_{H} \\
\mathrm{ml} / \mathrm{min}\end{array}$ & $\begin{array}{c}\dot{m}_{C} \\
\mathrm{ml} / \mathrm{min}\end{array}$ & $T_{H}\left({ }^{\circ} \mathrm{C}\right)$ & $T_{C}\left({ }^{\circ} \mathrm{C}\right)$ & $\mathrm{Re}$ \\
\hline 1 & 1.0 & 1.0 & 45 & 45 & 156.9 \\
\hline 2 & 2.0 & 1.0 & 62 & 12.5 & 312.1 \\
\hline 3 & 2.0 & 1.0 & 64 & 13 & 314.4 \\
\hline
\end{tabular}

\section{RESULTS AND DISCUSSION}

In this study an image processing analysis is performed on the infrared thermography images aimed at improving the quality of the acquired images so as to make it possible to obtain information in principle not provided by the raw image.

Two image processing techniques were applied to the three cases mentioned in the previous section: a Laplacian and a Gradient filter. Both filters have the function of detecting edges of the images (high pass filters). The Laplacian filter uses the second derivative of the image for this purpose while the Gradient filter uses the first derivative. By using these filters it is possible to enhance the edges of the thermographic images, which allows to identify more clearly the heat diffusion along the plate surface. Both filters were implemented using the Mathematica 7.0 platform, which provides a routine for the Laplacian filter called LaplacianFilter and another routine for the Gradient filter, called GradientFilter.

\section{Case 1}

Figures 11 a, b show the thermographic images at the initial condition, the transient state and steady state for Case 1 . In the three figures it can be seen two lines, one following the pixel column of number 300 and another approximately along the centerline of the micro-channel, on pixel line 236. These correspond to the pixel lines to be analyzed in more detail in the course of this work. In Figure 11a is shown the plate surface in its initial condition where it can be seen that the emissivity of the plate surface is fairly uniform and that the plate is in thermal equilibrium with the external environment. Figure $11 \mathrm{~b}$ is taken at time equal to 145 seconds and is typical of the transient regime of the experiment. Figure 11c is taken in time equal to 500 seconds, and represents the steady state of this experiment. Comparing the images shown in Figs. 11 a-c, there is a quite noticeable increase in the lateral spread of heat over the substrate.
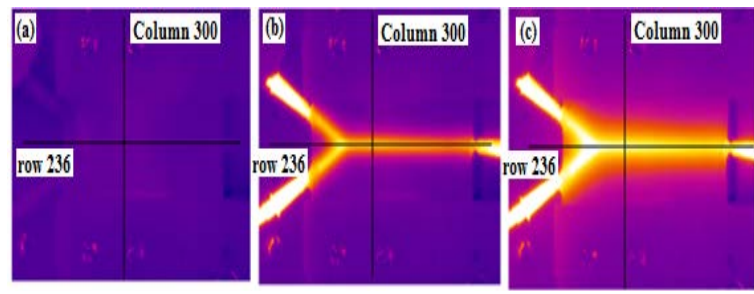

Figure 11. IR images in straight micro-mixer for (a) initial state, (b) transient state and (c) steady state.

Figure 12a shows the enlarged image of the mixing channel of Figure 11b, while Fig.12b further enlarges section A from the previous figure, where it is identified the pixels where the channel is located. In this case, the spacing calculated for each pixel was of $0.22 \mathrm{~mm}$ and the channel occupies approximately $2.0 \pm 0.5$ pixel lines of the image, resulting in a channel with dimensions of $0.44 \pm 0.11 \mathrm{~mm}$, which agrees closely to the measured value by microscopy that is approximately $0.450 \mathrm{~mm}$.
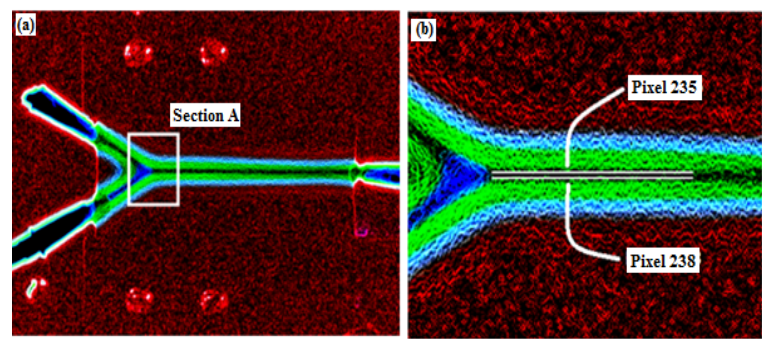

Figure 12. a) Enlarged image of the beginning of the mixing channel of Figure 11b;.b) enlarged Section A of figure 12.a (Case1).

Figures 13a,b present the temperature profiles along the pixel column 300, for different times from 50 s to 100 s with a step of 10 s, and from 200 s to 500 s with a step of $100 \mathrm{~s}$. Figure $13 \mathrm{~b}$ zooms on the region around the micro-channel, where it is possible to notice that the peak temperature occurs around the pixel line 236. Through these figures, one can clearly observe the temperature peaks inside the region of the 
hot fluid flow along the channel and the spreading of the temperature distribution due to lateral diffusion into the substrate with the progress of time.

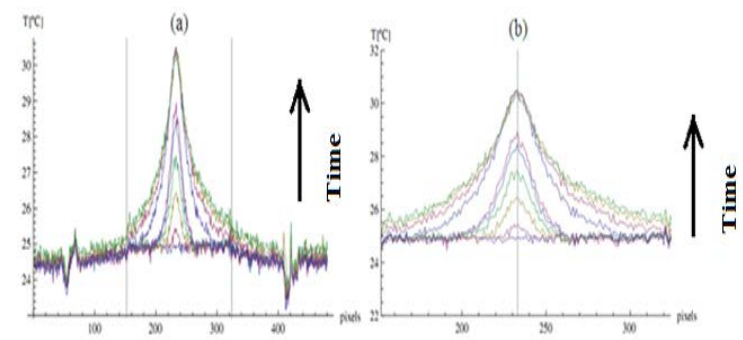

Figure 13. (a) Temperature distribution along the pixel column 300 and (b) zoom in the region around the micro-channel (Case1).

Figure 14 shows the temperature profile along the longitudinal length of the mixing channel, in the pixel lines 236, 237 and 238.

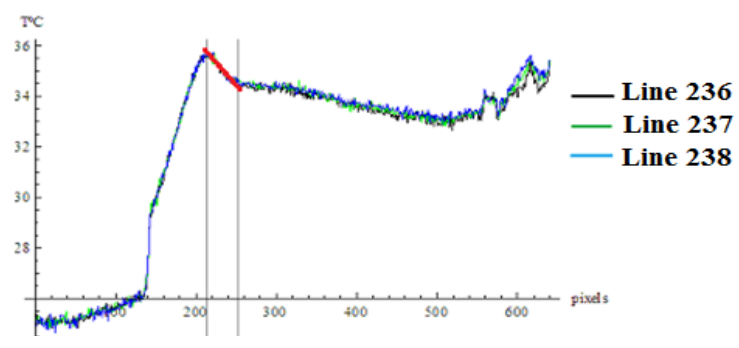

Figure 14. Longitudinal temperature distribution along the pixel lines 236, 237 and 238 for the image shown in Fig.11c (Case 1).

The linear region (red line), delimited by the vertical lines shown in Figure 14, allows estimating the Peclet number $(\mathrm{Pe})$ from its slope. The Peclet number was calculated at the center of the microchannel, in each row of pixels, from the dimensionless energy conservation equation:

$$
\mathrm{U}(\mathrm{R}) \frac{\mathrm{Pe}}{2} \frac{\partial \theta_{\mathrm{f}}}{\partial \varphi}=\nabla^{2} \theta_{\mathrm{f}}
$$

where the dimensionless velocity profile was assumed as $\mathrm{U}(\mathrm{R})=2\left(1-\mathrm{R}^{2}\right), \theta_{\mathrm{f}}$ is the dimensionless temperature of the fluid and $\varphi$ is the dimensionless longitudinal coordinate.

Assuming that the Peclet number is calculated by evaluating Eq.(1) at the center of the channel, $\mathrm{R}=0$, it takes the following form:

$$
\left.\operatorname{Pe} \frac{\partial \theta_{\mathrm{f}}}{\partial \varphi}\right|_{\mathrm{R}=0}=\left.\nabla^{2} \theta_{\mathrm{f}}\right|_{\mathrm{R}=0}
$$

The Peclet number estimate from Eq. (2) can then be obtained in the following form:

$$
\mathrm{Pe} \cong \frac{\left.\frac{\partial^{2} \mathrm{~T}_{\text {exp }}}{\partial \mathrm{y}^{2}}\right|_{\mathrm{y}=\text { row }} \frac{\mathrm{f}_{\mathrm{z}}}{\left.\frac{\partial \mathrm{T}_{\text {exp }}}{\partial \mathrm{z}}\right|_{\mathrm{y}=\text { row }}} \mathrm{f}_{\mathrm{y}}^{2}}{}
$$

where $\mathrm{T}_{\exp }$ is the experimental temperature distribution, $\mathrm{y}$ and $\mathrm{z}$ are the transversal and axial coordinates, respectively, and $\mathrm{f}_{\mathrm{y}}$ and $\mathrm{f}_{\mathrm{z}}$ is the spacing that each pixel occupies in the image in the transversal and axial directions, respectively.

The second derivative in the transversal direction, $\left.\frac{\partial^{2} \mathrm{~T}_{\exp }}{\partial \mathrm{y}^{2}}\right|_{\mathrm{y}=\text { row }}$, is evaluated at the center of the channel (pixel line at the channel center) and the first derivative, $\frac{\partial \mathrm{T}_{\mathrm{exp}}}{\partial \mathrm{z}}$, is the slope (red line) that appears in Figure 15.

For this case the Peclet number was estimated in the three pixel lines yielding an average value, $\mathrm{Pe}=$ $636.23 \pm 77.9$, while a pre-calculated value of $\mathrm{Pe}=639.8$ was obtained with the experimentally determined average velocity of $\mathrm{u}_{\mathrm{m}}=0.209 \mathrm{~m} / \mathrm{s}$.

\section{Case 2}

Figures 15a-c show the thermographic images related to the initial condition, transient state and steady state for the sinuous micro-mixer of Case 2. In these figures it can be seen two lines of pixels, one in the pixel column 345 and another on the pixel line 232, approximately in the middle of the inlet of the mixing channel. Figure 15a shows the plate surface at its initial condition and it can be observed the plate surface emissivity uniformity and the thermal equilibrium of the plate with the external environment. Figure $15 \mathrm{~b}$ is taken at 70 seconds and represents the transient regime of this experiment. Figure $15 \mathrm{c}$ is taken at 374 seconds and represents the steady state of the experiment. It can be observed from Figures 15b,c that within the transient regime one may still perceive the sinuous profile of the micro-channel, whereas in the steady state it is no longer possible to notice this sinuosity from this raw thermographic image, due to the heat diffusion across the substrate surface.
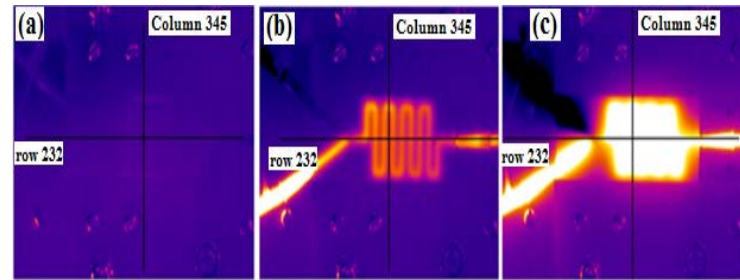

Figure 15. IR images in sinuous micro-mixer for (a) initial state, (b) transient state and (c) steady state. 
Figure 16a shows the image produced by the Gradient filter as applied to the image shown in Figure 15b. It can be observed that the image contours are sharper and allow to identify the size of the micro-channel in both the longitudinal and transversal orientations. It should be noted that the horizontal channels are wider than the vertical ones due to the position of the mask lines relative to the position of the lamps of the photoexposer during the manufacturing process of the micro-mixer. Figure $16 \mathrm{~b}$ shows the magnified image of a section of the mixing channel of Figure 16a, where it is possible to identify the pixel lines where the channel is located. The dimension of each pixel in the image is $0.21 \mathrm{~mm}$ in the horizontal coordinate and $0.25 \mathrm{~mm}$ in the transversal coordinate. The vertical channel occupies $1.5 \pm 0.5$ pixel rows in the image, resulting in a channel of $0.315 \pm 0.105 \mathrm{~mm}$, which agrees closely with the measured value in the microscope, 0.320 $\mathrm{mm}$. The horizontal channel also occupies $1.5 \pm 0.5$ pixel rows in the image resulting in a channel of width $0.375 \pm 0.125 \mathrm{~mm}$, which also approaches the value obtained through microscopy for this channel, $0.290 \mathrm{~mm}$
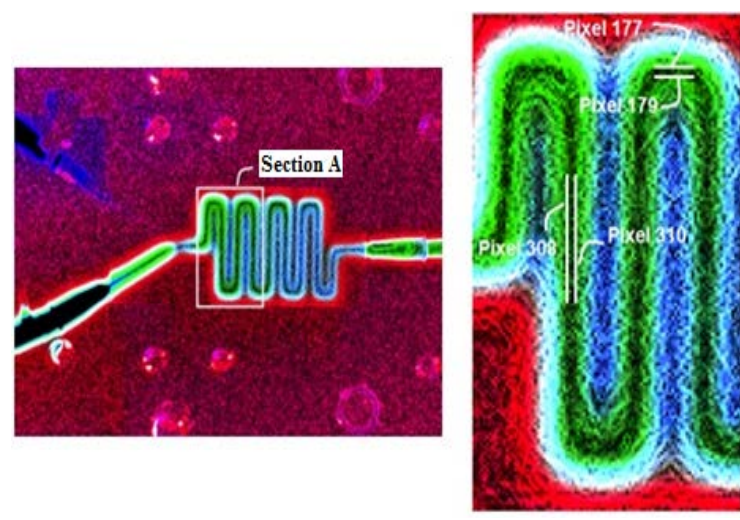

Figure 16. a) Filtered image of Fig.9b with the gradient filter (Case 2); b) Enlarged image of section A of Fig. 16a.

Figures 17a,b show the temperature profiles along the pixel column 345, for different time values from 50 s to 100 s with a step of 10 s and from 200 s to 500 s with a step of $100 \mathrm{~s}$. Figure $17 \mathrm{~b}$ shows the enlarged graphs of the plate region bounded by the vertical lines shown in Figure 17a, where it is possible to notice a peak temperature (identified by the vertical line shown in Figure 17.b), which corresponds to crossing the region of a horizontal channel section present in the corresponding longitudinal pixel line. In Figure 17a the vertical lines that appear correspond to the region that delimits the plate containing the micro-mixer. Through these figures one can clearly observe the temperature peaks related to the region where the micro-channel is located and the increase of the temperature values due to diffusion across the substrate with the progress of time.
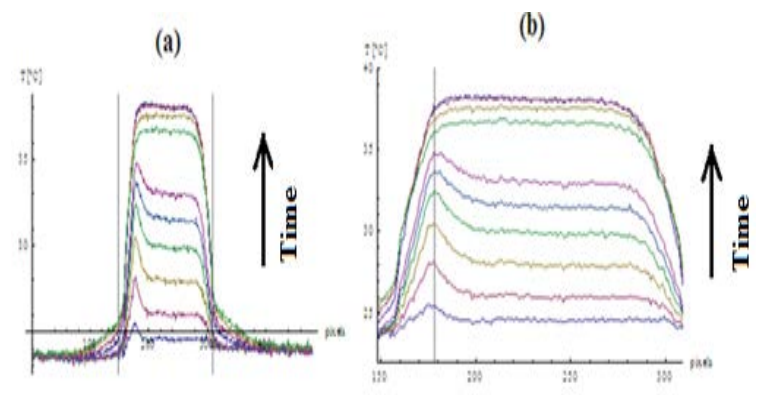

Figure 17. a) Temperature distribution along the column pixel 345; b) enlarged region delimited by the vertical lines shown in Fig. 17a (Case 2).

Figure 18 shows the longitudinal temperature profile along the mixing channel length, at the pixel line 232. One can observe various temperature peaks that correspond to crossing of the eight vertical channels along this longitudinal pixel line. The vertical lines that appear in the figure correspond to the region that delimits the plate containing the micro-mixer.

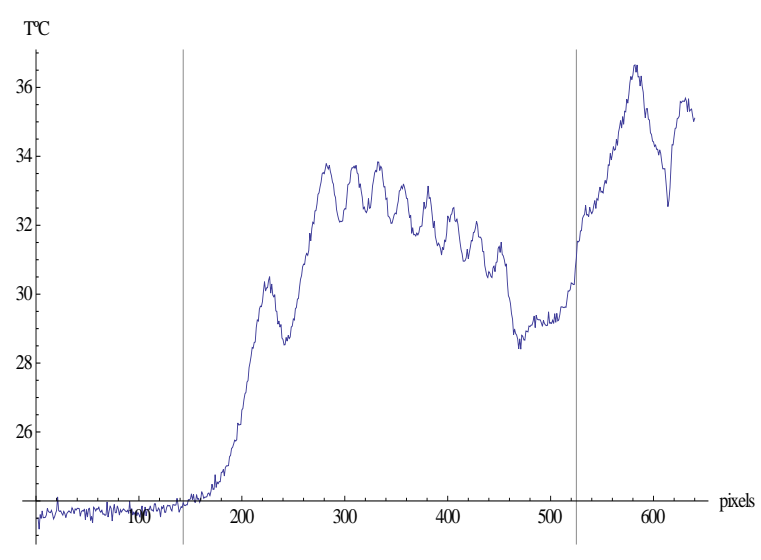

Figure 18. Longitudinal temperature distribution along the pixel line 232 for the image in Fig. 15b.

\section{Case 3}

Figures 19a-c show the thermography images for the initial condition, the transient state and the steady state for the zig-zag micro-mixer of Case 3 . Two lines were plotted on these figures, one at the pixel column 231 and another approximately at the middle of the inlet micro-channel on the pixel line 238. Figure 19a shows the initial condition of the surface of the plate, where it can be seen that the emissivity of the plate surface is fairly uniform and that the plate is in thermal equilibrium with the surrounding environment. Figure $19 \mathrm{~b}$ is taken at time equal to 85 seconds and is typical of the transient regime of the experiment. Figure $19 \mathrm{c}$ is taken at time 431 seconds, and represents the steady state of this experiment. In Figures 19b,c it is observed that the lateral heat diffusion markedly increases the temperature in the whole plate surface over time. 

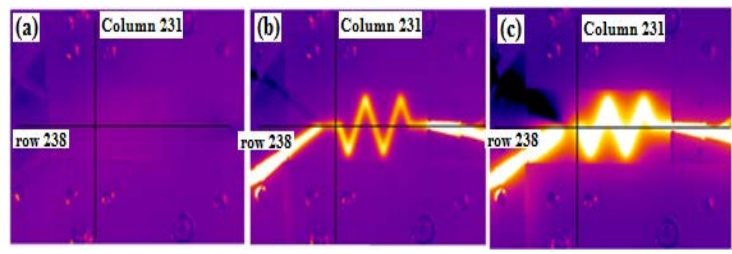

Figure 19. IR images in the zig-zag micro-mixer for (a) initial state, (b) transient state, (c) steady state.

Figure 20a shows the resulting image from application of the Gradient filter as obtained from the thermographic image in Fig. 19b. In this image it is possible to observe the details of the micro-mixer contours and note that it is possible to identify the size of the micro-channel at different positions. Figure 20b shows a magnified image of a section of the mixing channel of Figure 20a, where it is possible to identify the pixel lines where the channel is located. In this case, the width of the micro-channel is calculated at the input of the micro-mixer. The spacing calculated for each pixel was $0.21 \mathrm{~mm}$ in the horizontal direction and $0.27 \mathrm{~mm}$ in the vertical direction. The channel occupies approximately $1.5 \pm$ 0.5 lines of pixels in the image, resulting in a channel with width of $0.405 \mathrm{~mm} \pm 0.135 \mathrm{~mm}$, which compares still reasonably to the measured value, which is approximately $0.310 \mathrm{~mm}$.

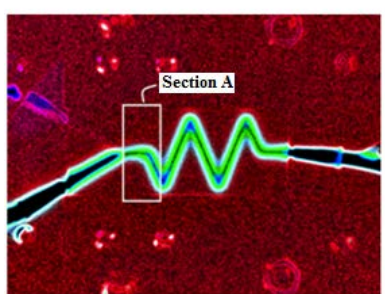

(a)

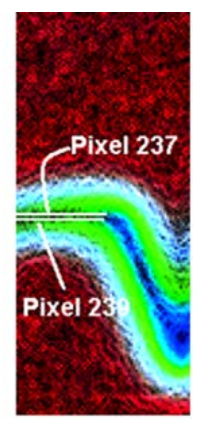

(b)
Figure 20. a) Filtered image of Fig. 19b with the gradient filter (Case 3); b) Enlarged image of section A of Fig. 20a.

Figures 21a,b present the temperature profiles along the pixel column 231, for different times, from 50 s to 100 s with a step of 10 s and from 200 s to 500 s with a step of 100s. Figure 21b shows the magnified region of the plate bounded by the vertical lines shown in Figure 21a, where it is noticed that the peak temperature occurs around the pixel line 238 (vertical line shown in Figure 21b), which crosses the microchannel. In Figure 21a the vertical lines correspond to the region that delimits the plate containing the micro-mixer. Through these figures, one can clearly see the peak temperature within the region of hot fluid flow along the channel and the increasing of the temperature distribution due to lateral diffusion into the substrate with the progress of time.

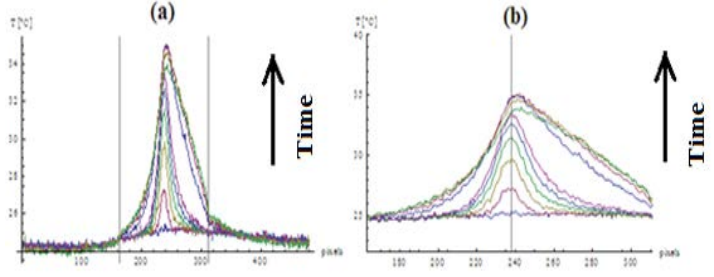

Figure 21. a) Temperature distribution along the pixel column 345; b) enlarged region around the micro-channel (Case 3).

Figure 22 shows the longitudinal temperature profile along the length of mixing, at the pixel line 238. One can observe the several temperature peaks that correspond to the regions where the diagonal channels are crossed by the longitudinal pixel line. The vertical lines that appear in the figure correspond to the region that delimits the plate containing the micro-mixer.

\section{CONCLUSIONS}

Through the images provided by infrared thermography it was possible to perform an image processing analysis in order to obtain qualitative and quantitative information about the micro-mixers thermal behavior. Two filters were applied (Gradient and Laplacian filters) so as to emphasize the edges of the micro-system and identify the regions of heat diffusion in the substrate. The application of these filters also allows for estimating the Peclet number of the flow, and therefore, the average velocity in the channel, critically compared with the direct assessments from control of the flow in each stream.

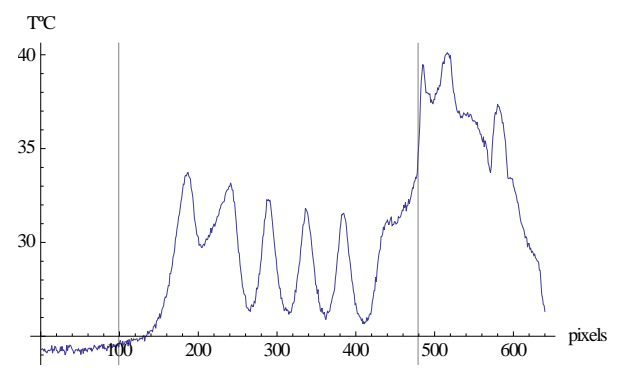

Figure 22. Longitudinal temperature distribution along the pixel line 238 for the image in Fig. 19b.

\section{ACKNOWLEDGEMENTS}

The authors would like to acknowledge the financial support provided by the Brazilian (CNPq, FAPERJ, and CAPES) and French (CNRS) agencies.

\section{REFERENCES}

Engler, M., Kockmann, N., Kiefer, T., and Woias, P., 2004, Numerical and Experimental Investigations on Liquid Mixing in Static 
Micromixers, Chemical Engineering Journal, Vol. 101, pp. 315-322.

Fernandes, J. C. B., and Ferreira, L. O. S., 2006, Manufacturing of Miniature Fluidic Modules for Labon-a-Chip Using UA Photoresin form Flexographic Platemaking Process, Journal of the Brazilian Chemical Society, Vol. 17, No 4, pp. 643-647.

Fonseca A., 2008, Construção e Avaliação de Microssistemas para Análise em Flux, Doctoral Thesis, Universidade de Campinas, Campinas, SP. (in Portuguese)

Pohar, A., and Plazl, I., 2009, Process Intensification through Microreactor Application, Chem. Biochem. Eng., Vol. 23, pp. 537-544.

Pradere, C., Joanicot, M., Batsale, J. C., Toutain, J., and Gourdon, C., 2004, Processing of Temperature Field in Chemical Microreactors with InfraRed Thermography, Quantitative InfraRed Thermography Journal, Vol. 1, pp.5-20.

Tabeling, P., 2003, Introduction à la Microfluidique, Collection èchelles, Belin.

Xu, B., Wong, T. N., Nguyen, N. T., Che, Z., and Chai, J. C. K., 2010, Thermal Mixing of two Miscible Fluids in a T-Shaped Microchannel, Biomicrofluidics, Vol. 4, pp. 044102-1 - 044102-12. 